\title{
Simulation and optimization of an optical-electrical combination conductor system
}

\author{
B. Lustermann ${ }^{1}$, M.Viehmann ${ }^{1}$, E. Manske ${ }^{2}$, U. Gerhardt ${ }^{2}$, C. Kloß ${ }^{1}$ \\ ${ }^{1}$ Nordhausen University of Applied Sciences, Weinberghof 4, 99734 Nordhausen, Germany, \\ lustermann@fh-nordhausen.de \\ 2 Ilmenau University of Technology, PO Box 100 565, 98684 IImenau, Germany
}

\begin{abstract}
:
Over the past few years the innovative optical-electrical combination conductor system CONDUS has been developed and investigated at the Nordhausen University of Applied Sciences. This invention is patented in many countries and opens completely new fields for applications [1].

The principal item of the optical-electrical system CONDUS is a combination of an electrical conductor (e.g. a copper wire) with a coat of an optical polymer (see Figs. 1 und 2). The optical cover of the electric wire enables the immediate detection of arc faults in a single structural component.

The aim of this work is to present a simulation model for such conductors and to use it in order to investigate the influence of different designs, materials and geometries. In particular, the influence of surface roughness and volume scattering of optical materials is studied and compared to experimental data.
\end{abstract}

Key words: optical-electrical combination conductor system, polymer optical fiber, ray-tracing, arc fault detection

\section{Introduction}

The detection of electrical arcs is an increasing problem in different applications such as electro mobility and renewable energy technology. Loose and aging cables of electric circuits with a voltage of more than $12 \mathrm{~V}$ can induce an arc fault [2]. The challenge is to detect the arc fault, before it can destroy the whole equipment. There are different solutions for arc detection and protection systems in the high voltage sector [3]. For low-voltage applications (max. $600 \mathrm{~V}, 35 \mathrm{~A})$ several electrical solutions exist [4]. All these solutions need a complex signal processing. With an optical coat of the electrical cable it would be possible to detect the light of the arc fault in the fastest way. Such a transparent coating of a flexible cable must have low losses, good bending properties and high temperature stability. To optimize the structure of the optical-electrical combination conductor system, the thickness of the optical cladding and core layers as well as the arrangement of the optical receivers it is necessary to have an appropriate optical model for the tubular optical fiber.

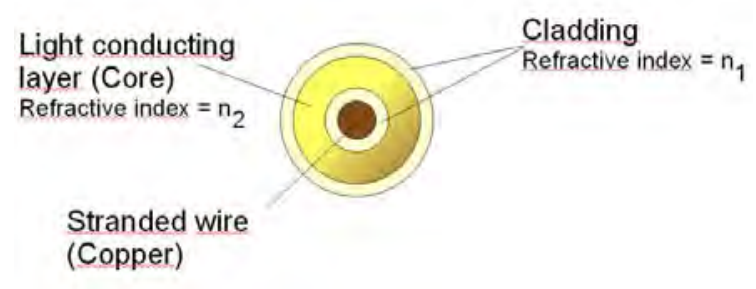

Fig. 1. Top view of the optical-electrical combination conductor system
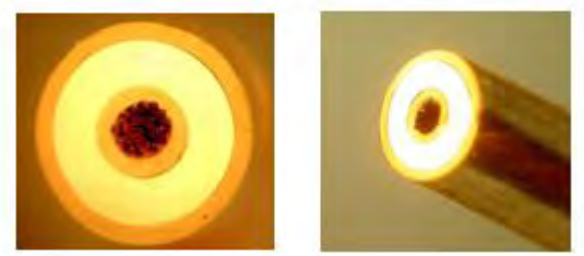

Fig. 2. Top and side view of prototype (diameter of wire $=0.9 \mathrm{~mm}$; cladding thickness $=0.3 \mathrm{~mm}$; core thickness $=0.8 \mathrm{~mm}$ ) 
There are, at least, two applications for opticalelectrical combination conductor systems:

a) The detection of parallel arc faults by guiding of light to the optical receiving unit (see Fig. 3). Due to the stochastic nature of the arc fault, the conditions of the coupling process are difficult to quantify and to calculate. If the optical coat serving as isolation material of such a conductor has a mechanical defect or the electric field strength exceeds a certain limit, an arc fault can occur. The heat the will destroy or melt the polymer material. Test measurements show, nevertheless, that the period during which the boundary surface remains transparent suffices to detect the arc. The spectral distribution of an arc depends on the involved materials, the intensity depends on the dielectric strength (see Fig. 4).

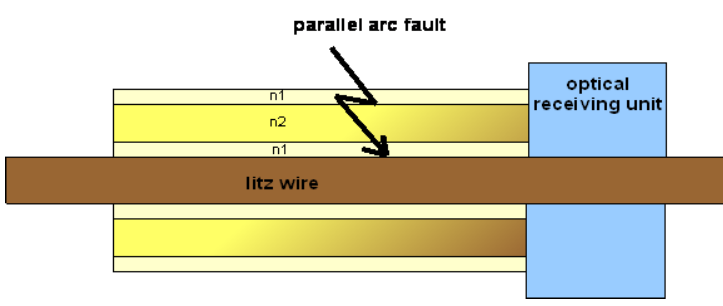

Fig. 3. Detection of a parallel arc fault

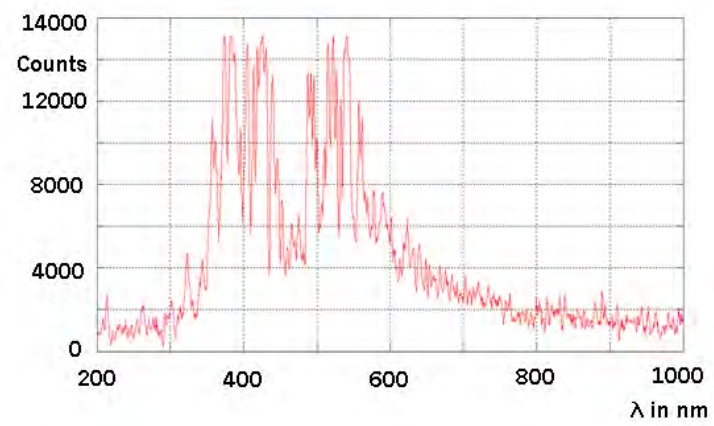

Fig. 4. Arc fault (air-copper-stainless steel; $30 \mathrm{~V}$ )

Fig. 5 shows the propagation of light coupled into a tubular waveguide. In dependence on the thickness of the electrical wire or cable, at short distances the light will not show a uniform distribution around the circular end face. A circular detector array is necessary for sure arc detection.

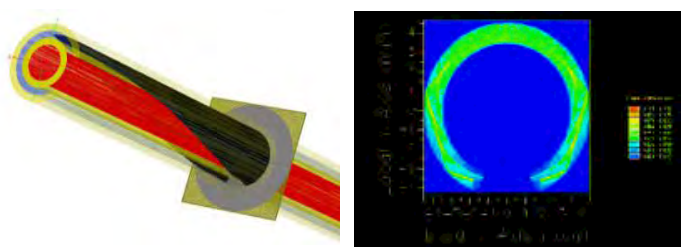

Fig. 5. Light propagation in the absence of scattering effects b) The second idea is to monitor the optical coating in order to detect isolation defects (see Fig. 6). Fig. 7 shows first modules with circularly arranged laser diodes and photo diodes achieving this goal.

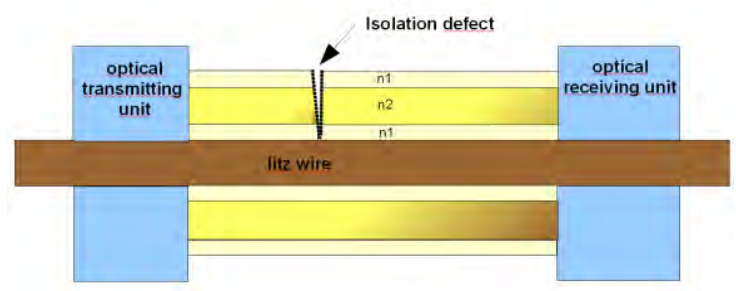

Fig. 6. Monitoring with laser diodes

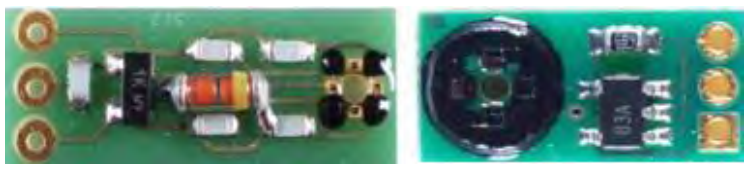

Fig. 7. Circular transmitter (left) and receiver (right)

\section{Polymer optical fiber}

The problem of modeling polymer optical fibers (POF) lies in the material structure and the scattering properties of polymers. The intrinsic and extrinsic losses in step index (SI) or multistep index (MSI) polymer optical fibers are described in Refs. [5,6,7]. Scattering in POF has been intensively investigated, because there are many different processes causing large optical power losses $[9,10]$.

Ray tracing software can be used to investigate multimode fibers [11]. A lot of issues can be faced: the positioning of the optical transmitter or receiver, the influence of the numerical aperture, bending losses, wavelength dependences of refractive indexes and absorption coefficients. While performing simulations, the user has to ensure two things:

- The limitations of geometrical optics should not be violated.

- The numerical resolution must be high enough.
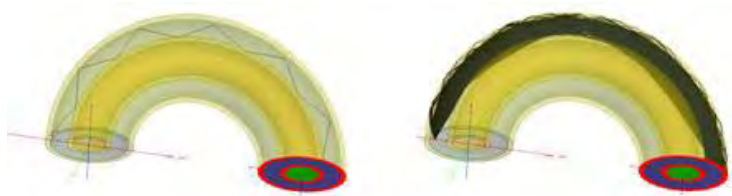

Fig. 8. Bended optical-electrical conductor

Simple investigations of straight or bended fibers (see Fig. 8) without any scattering effects show that the transmitted power increases with the radius of the electrical wire or of the inner cladding layer. This is caused by the increase 
of the angle of total reflection. The simultaneous increase of the optical path length (dispersion) is not important for the aim of detecting arc faults or isolation defects. However, the development of an opticalelectrical conductor system for data transmission is also imaginable.

In tubular optical fibers the following effects contribute to the scattering of light:

- the roughness of the front and end faces of the fiber,

- the volume scattering caused by inhomogeneities,

- the roughness of the core-cladding boundary,

- the roughness of the outer cladding layer, and

- the roughness and the structure of the inner cladding-copper boundary.

In order to model these effects we applied different scattering models for the different scales of disorder: on the one hand smooth surfaces (usually the core-cladding boundary) with impurities of size $<100 \mathrm{~nm}$, on the other hand the rather rough surfaces at the front and end faces with rms roughnesses of up to 80 $\mu \mathrm{m}$.

\section{Volume scattering}

The behaviour of an incident electromagnetic wave interacting with a scatter particle depends on the relation between the wavelength and the particle size as well as on particle properties. Volume scattering is a stochastic process; the change of propagation direction can be described by an angular distribution function. The volume scatter model which is used in the ray-tracing software FRED calculates this angular distribution by the Henyey-Greenstein formula [15]:

$p(\theta)=\frac{1}{4 \pi} \frac{1-g^{2}}{\left(1+g^{2}-2 g \cos \theta\right)^{3 / 2}}$.

Here, the scattering anisotropy parameter $g$ (which is the averaged cosine of the direction of scattered rays) varies in the range $-1 \leq g \leq+1$; $g=-1$ corresponding to total backward scattering, $g=0$ to isotropic (Rayleigh) scattering and $g=+1$ to total forward scattering (Mie scattering for large particles). Furthermore, a scattering coefficient $\mu_{s}$ must be known, which describes the average distance between two scattering events. The experimental determination of these parameters is expensive, examples are described in Refs. $[13,14]$.

\section{Front and end face roughness}

Fig. 9 shows a front face surface from a test polymer fiber with a core diameter $d_{\text {Core }}=8 \mathrm{~mm}$, the core material being silicone, the cladding material - FEP. The front face is opaque. However, the measured transmission of the uncut core material ( $6 \mathrm{~mm}$ thickness) is well above $90 \%$.

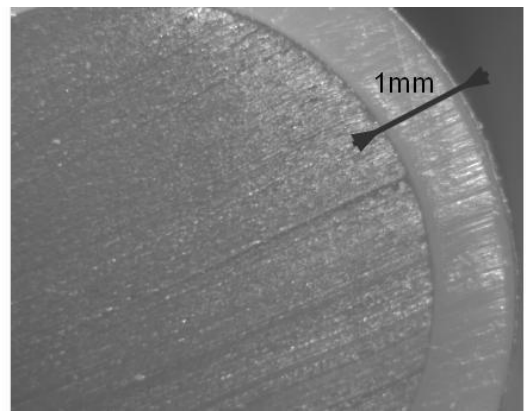

Fig. 9. Front face of a silicone fiber

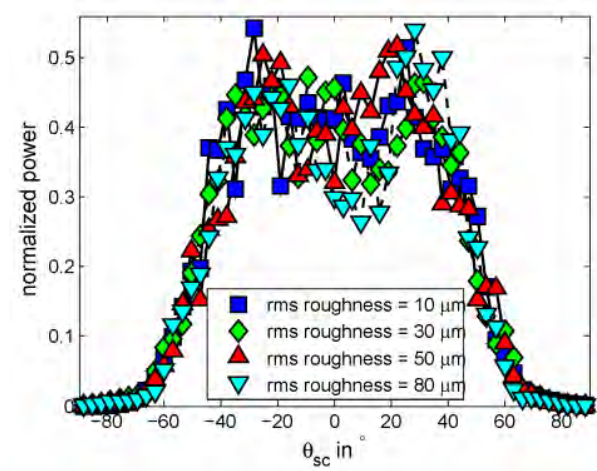

Fig.10. Angular distribution in the core caused by the scattering at the front face (simulation)

Fig. 10 shows the intensity spread function behind the front face illuminated by a laser. The figure was generated by a simulation using a non-isotropic, stochastic rough front surface, the rms roughness ranging from $10 \mu \mathrm{m}$ to 80 $\mu \mathrm{m}$ in order to simulate the grooves.

The large scatter angles cause large intensity losses since the light will be decoupled immediately. To circumvent this problem, the transmitter can be moulded into the material or the moulded transmitter can be pressed onto the front face.

\section{Roughness of the core-cladding boundary}

The roughness of a surface can be quantified by the root mean square (rms) $\sigma$ of the height profile. Because the rms roughness depends on the sampling resolution, the surface profile function is sensitive to the instrument used for scanning. Especially for the determination of the autocovariance function of the surface (and therefore the correlation length $\tau$ ) the scan resolution must be high enough. 
Roughness measurements were performed at IImenau University of Technology with the nano positioning and measuring machine NMM1 with a laser focus sensor $[18,19]$. The height resolution is better than $1 \mathrm{~nm}$ and the lateral resolution is limited by the laser spot diameter $(<1 \mu \mathrm{m})$. For a silicone fiber we get an rms roughness of $\sigma=63 \mathrm{~nm}$. The scan length was $1.25 \mu \mathrm{m}$, unfortunately not small enough to compute the correlation length (see Fig. 11).

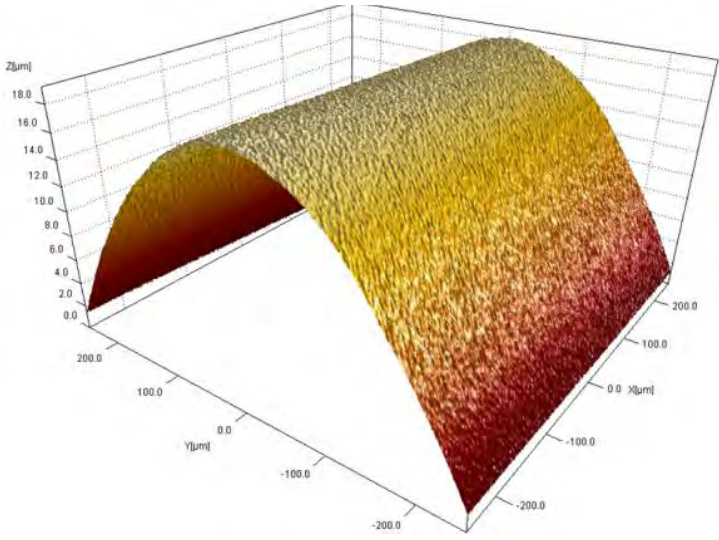

Fig. 11. Surface scan of the silicone core without cladding

The quotient of the rms roughness $\sigma$ and the correlation length $\tau$ is denoted as slope; it crucially determines the surface geometry. For the numerical simulation we assume that $\tau$ is of the same order as $\sigma(0.5<$ slope < 2$)$. It turns out, that this assumption is sufficient to achieve good agreement with measured data.

\section{Surface statistics and Power Spectral Density (PSD)}

In a ray tracing software directions of scattered rays are computed from the Bidirectional Scatter Distribution Function (BSDF). The best way to create the BSDF is to measure the scattered light with a scatterometer. If this is not possible, the 1D- (in the case of isotropic surfaces) or 2D-scanned profile can be used to generate the spectral distribution function.

The Power Spectral Density (PSD) is defined by the Fourier transform of the height profile $h$. For isotropic surfaces the information is contained in the 1-dimensional PSD

$S_{1}(f)=\lim _{L \rightarrow \infty} \frac{2}{L}\left|\int_{-L / 2}^{L / 2} e^{2 \pi i f x} h(x) \mathrm{d} x\right|^{2}$.

The simulation of a rough, isotropic surface starts, therefore, from the generation of a random, but correlated, $h(x)$ [20]. For our simulation, we generated (for each combination of $\mathrm{rms}$ roughnesses $(10 . .100 \mathrm{~nm})$ and slopes $(0.5,1,2)) 100$ surfaces and calculated the average 1D-PSD. In order to smooth the resulting data, we used the $A B C$-correlation function suggested in Ref. [17]: $S_{1}(f)=\frac{A}{2\left[1+(B f)^{2}\right]^{C / 2}}$

This ansatz has both the advantage of almost perfectly fitting the data (see Fig. 12) and of leading to an analytic expression for the 2DPSD (being a certain integral transform of $S_{1}$ ):

$$
S_{2}(f)=\frac{K}{2\left[1+(B f)^{2}\right]^{(C+1) / 2}}, \quad K=\frac{A B}{2 \sqrt{\pi}} \frac{\Gamma\left(\frac{C+1}{2}\right)}{\Gamma\left(\frac{C}{2}\right)}
$$

Finally, from $S_{2}(f)$ the Bidirectional Scatter Distribution Function can be calculated, for details see Ref. [17].

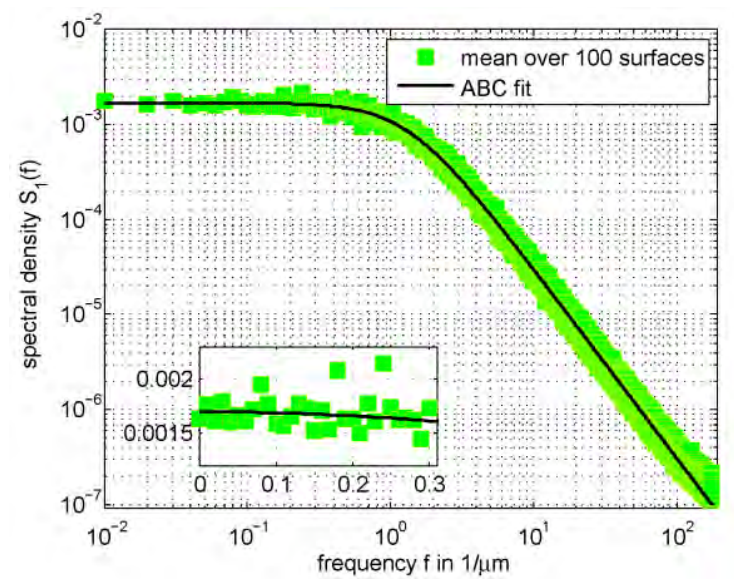

Fig. 12. Spectral density $S_{1}(f), L=100 \mu m, \sigma=60$ $\mathrm{nm}$, slope $=0.5$

\section{Comparison to experimental data}

In order to validate the simulations described above we confronted them to the bare silicone test fiber scanned with the NMM1. The core diameter was equal to $3.54 \mathrm{~mm}$ and the wavelength was $660 \mathrm{~nm}$.

At this wavelength, the absorption coefficient of silicone equals $\alpha_{a b s}=2.763 * 10^{-5} \mathrm{~mm}^{-1}$ (corresponding to $D_{a b s}=0.0012 \mathrm{~dB} / \mathrm{cm}$ [12]). The volume scattering was modeled by the Henyey-Greenstein scatter model with $g=0.8$ and $\mu_{s}=0.18 \mathrm{~m}^{-1}$. These values correspond to a volume attenuation $D_{V \text {-scat }}=0.003 \mathrm{~dB} / \mathrm{cm}$ for a core diameter of $1 \mathrm{~mm}$ [12].

Note that for simulation we located both the light source and the detector surface inside the fiber (in order to exclude the losses connected with front and end face).

By measuring the outcoming power at different length (cutting the original fiber step by step), the losses due to in- and outcoupling effects can be separated from the attenuation inside 
the fiber. The resulting attenuation coefficient was found to be $\alpha_{\text {fiber }}=1.16 \mathrm{~m}^{-1}$ (see Fig. 14). From this value an attenuation factor $D=0.05$ $\mathrm{dB} / \mathrm{cm}(660 \mathrm{~nm})$ follows. The error caused by the relatively short length of the fiber is negligible, because the aperture of the fiber is high enough to guide all light in the fiber.

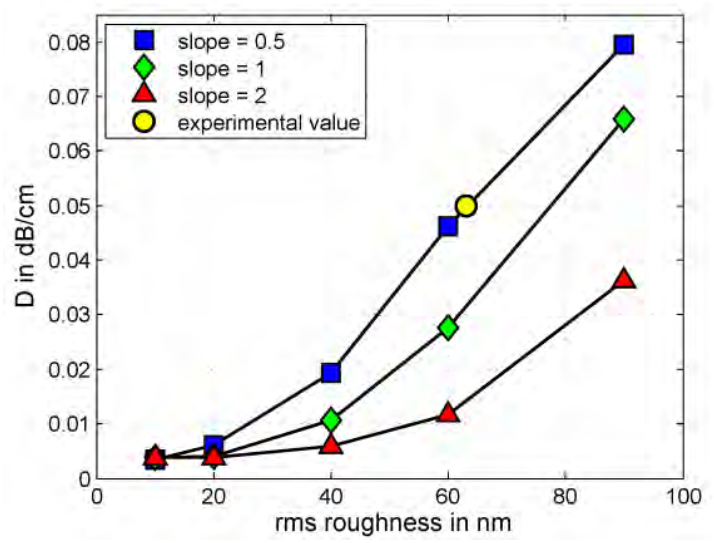

Fig. 13. Simulation results, bare silicone fiber without cladding, length $L=35 \mathrm{~cm}, d_{\text {core }}=3.54 \mathrm{~mm}$, including volume scattering $\left(g=0.8 ; \mu_{s}=0.18 \mathrm{~m}^{-1}\right)$

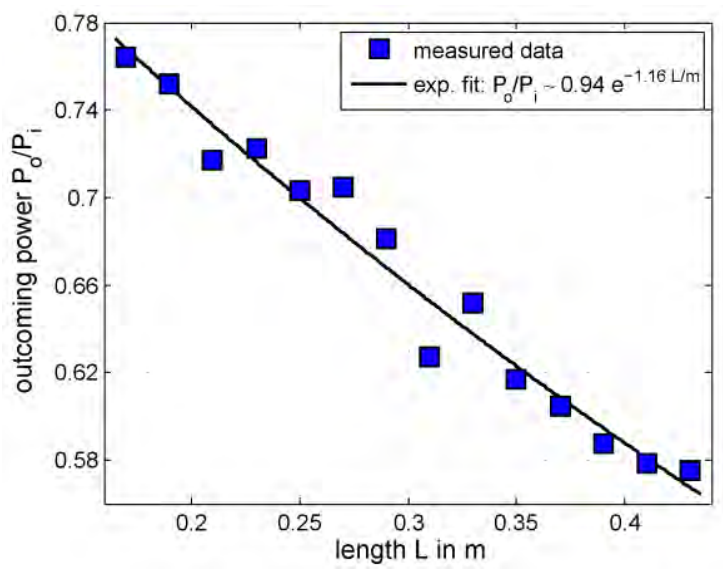

Fig.14. Bare silicone fiber $d_{\text {Core }}=3.54 \mathrm{~mm}$

From this value, combined with the measured $\mathrm{rms}$ roughness of $63 \mathrm{~nm}$, a value of the slope $\sigma$ / $\tau=0.5$ can be derived (see Fig. 13).

Therefore, we used for all simulations with silicone material the PSD-scatter-model calculated from surfaces with an rms roughness $\sigma=63 \mathrm{~nm}$ and slope $=0.5$.

Since these values are caused by the manufacturing process of the silicone fiber we can assume that the cladding layer has the same surface properties. If it would be possible to reduce the rms roughness to less than 20 $\mathrm{nm}$, the attenuation factor would go down to values comparable to the limitations caused by volume scattering and absorption.

\section{Results for tubular fibers}

Fig. 15 shows the results of simulations for several tubular geometries. With increasing radius of the electrical wire, $r_{\text {wire }}$, the attenuation factor is decreasing (due to the increase of the angle of acceptance for total reflection). At the same time, with decreasing core thickness the attenuation factor caused by surface scattering is increasing.

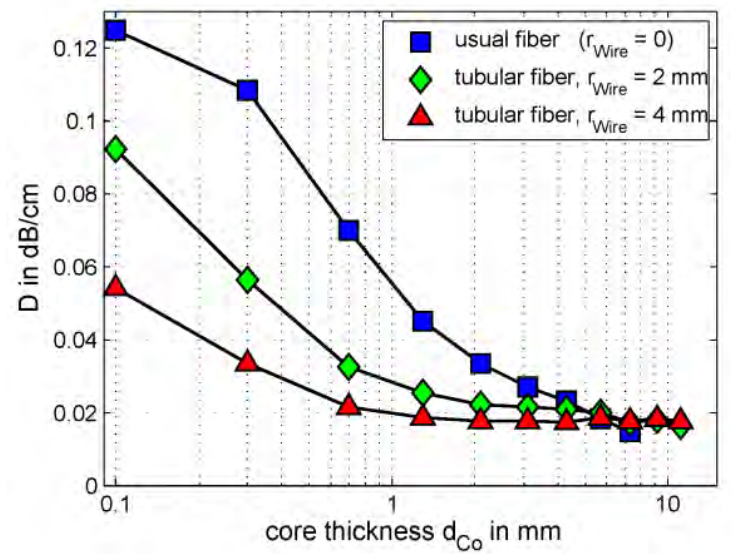

Fig.15. Attenuation of tubular fiber (inner and outer cladding thickness $=0.3 \mathrm{~mm}$ ) and usual step index fiber (cladding thickness $=0.3 \mathrm{~mm}$ )

In Fig. 16 the bending behaviour of a tubular fiber is shown. With decreasing bending radius the attenuation factor increases, since the condition for total reflection is violated by more and more rays. Moreover, although tubular fibers with small core thickness have a greater number of reflections, it turns out that their bending losses are smaller.

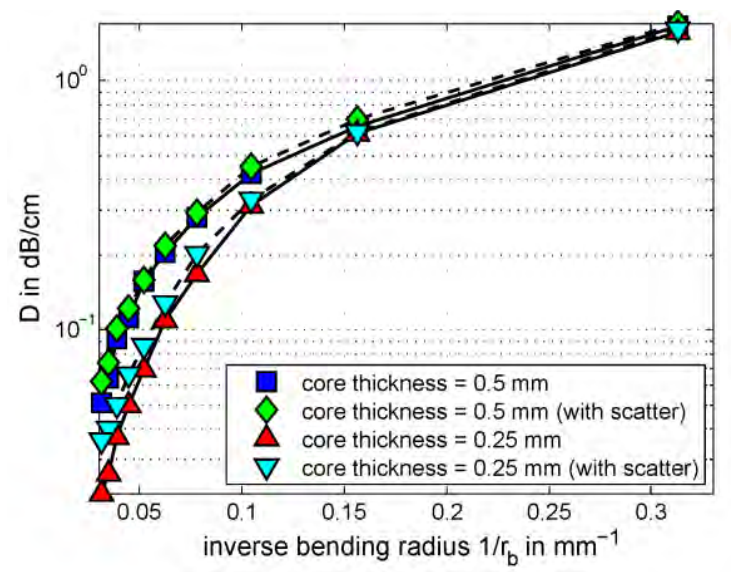

Fig.16. Bended tubular fiber with different core thicknesses (inner and outer cladding thickness $=0.3$ $\mathrm{mm}$ )

The developed model can be used to perform detailed investigations of the characteristics of the optical-electrical combination conductor system CONDUS. 


\section{References}

[1] M. Viehmann, Vehicle power supply cable with optical jacket monitoring and arcing interference detection. ATZelektronik, Heft 05/2012 (September 2012)

[2] P. Meckler, Störlichtbögen in AutomotiveBordnetzen: Fehlerquelle mit Brandgefahr, Elektronik Informationen 6 (2012)

[3] A. Klaus, Optische Störlichtbogendetektion in luftisolierten Mittelspannungsanlagen bei Störlichtbögen, Dissertation, TU IImenau (2006)

[4] E-T-A Elektronische Apparate $\mathrm{GmbH}$, Gefährliches Plasma: Lichtbogenerkennung in PV-Anlagen, messtec drivers Automation Vol.6, p.40-41 (2011)

[5] G. Aldabaldetreku, G. Durana, J. Zubia, J. Arrue, Analysis of intrinsic coupling loss in multi-step index optical fibres, OPTICS EXPRESS Vol.13, No. 06, 3283 (2005)

[6] G. Aldabaldetreku, I. Bikandi, M. A. Illarramendi, G. Durana, J. Zubia, A comprehensive analysis of scattering in polymer optical fibers, OPTICS EXPRESS Vol.18, No.24, 24536 (2010)

[7] J. T. Remillard, M. P. Everson, and W. H. Weber, Loss mechanisms in optical light pipes, APPLIED OPTICS Vol. 31, No. 34 (1992)

[8] K.Tang, R. O. Buckius, A statistical model of wave scattering from random rough surfaces; International Journal of Heat and Mass Transfer 44 (2001) 4059-4073

[9] C.-A. Bunge, H. Poisel, POF Modelling: Theory, Measurement and Application, Summary of the 1st International POF-Modelling Workshop 2007, Books on Demand $\mathrm{GmbH}, 2007$

[10] C.-A. Bunge, R. Kruglov, H.Poisel, Rayleigh and Mie Scattering in Polymer Optical Fibers; Journal of Lightwave Technology, Vol.24, No.8, 2006

[11] D. Cai, Optical and Mechanical Aspects on Polysiloxane Based Electrical-Optical-CircuitsBoards; http://d-nb.info/997586249/34; 2008

[12] G. Zeidler, Elastomere Optische Fasern (EOF), Foliensammlung, ITG-FG Treffen Oldenburg, 25./26.03.2003

[13] O. Pernell, Optische Untersuchungen zum Farbeindruck geschichteter dentaler Komposit füllmaterialien; AusDruck Berlin - Schaare \& Schaare GbR, Berlin 2008; online:

http://www.ausdruckberlin.de/fileadmin/user upload/DownloadDateien/veroeffentlicht/pernell1208.pdf

[14] S. Willmann, Bestimmung optischer Konstanten zur Beschreibung der Laserlicht-Gewebe Wechselwirkung; Diplomarbeit 1996; http://www.ilm.uni-duesseldorf.de/hering/ Tissue Group/DA Willmann.pdf
[15] Photon Engineering, FRED User Manual

[16] Photon Engineering, Stray Light Short Course 2011, Course Manual

[17] J. E. Harvey, N. Coi, A. Krywonos, Calculating BRDFs from Surface PSDs for Moderately Rough Optical Surfaces, Proc. of SPIE Vol.7426, 742601, 2009

[18] E. Manske, R. Mastylo, T. Machleidt, K.-H. Franke, G. Jäger, New applications of the nanopositioning and nanomeasuring machine by using advanced tactile and nontactile probes, In: Measurement Science and Technology, Vol. 18, pp. 520-527, 2007

[19] E. Manske, G. Jäger, T. Hausotte, R. FüßI, Recent developments and challenges of nanopositioning and nanomeasuring technology. In: Measurement Science and Technology, Vol. 23, 074001 (10 pp), 2012

[20] M. Deserno, How to generate exponentially correlated Gaussian random numbers, http://www.google.de/url?sa=t\&rct=j\&q=\&es rc=s\&source=web\&cd=1\&ved=0CDMQFiAA \&url=http $\% 3 \mathrm{~A} \% 2 \mathrm{~F} \% 2 \mathrm{Fwww.cmu}$. edu $\% 2 \mathrm{Fbi}$ olphys $\% 2 \mathrm{Fdeserno} \% 2 \mathrm{Fpdf} \% 2 \mathrm{Fcorr}$ gaussi an random.pdf\&ei=fclKUcyfD4zSsgat IGA BA\&usg=AFQjCNHIvInOfmdCfrY9vlEjBIQ9MU8rw 